\title{
Prevalencia e identificación genotípica de Enterococos Vancomicina resistentes en pacientes en un medio hospitalario
}

\author{
Ana V. Salas-Vargas ', Ricardo Boza-Cordero ${ }^{2}$, Warner Bustamante-García ${ }^{3}$, \\ Fernando García-Santamaría ${ }^{4}$, Edith Barrantes-Valverde ${ }^{5}$
}

\section{Re sum e n}

I Médico Internista. Sección de Medicina, Hospital San Juan de Dios.

2 Médico Infectólogo. Servicio de Infectología, Hospital San Juan de Dios, Profesor Asociado Escuela de Medicina, Universidad de Costa Rica.

${ }^{3}$ Microbiólogo. Laboratorio Clínico Hospital de Turrialba.

${ }^{4}$ Microbiólogo Catedrático, Centro de Investigación en Enfermedades Tropicales, Facultad de Microbiología, Universidad de Costa Rica.

5 Microbióloga, Jefe Laboratorio de Bacteriología, Hospital San Juan de Dios, Profesora Escuela de Medicina, Universidad de Costa Rica.

Abreviaturas: $\mathrm{UCl}$; unidad de cuidado intensivo; EVR, enterococos vancomicina resistentes; $\mathrm{CIM}$, concentraciones inhibitorias mínimas; EVS, enterococos vancomicina sensibles.

Correspondencia: Dr. Ricardo Boza Cordero Servicio de Infectología HSJD, Costa Rica Fax 258 2989. E-mail ricardob49@hotmail.com

ISSN 0001-6002/2004/46/1/19-26 Acta Médica Costarricense,(C2004

Colegio de Médicos y Cirujanos
Justificación y Objetivos: los enterococos son cocos Gram positivos y las especies de importancia médica son Enterococcus faecalis y E. faecium como productores de la mayoría de las infecciones en el ser humano y E.casseliflavus, E. gallinarum, E. durans, E.hirae, E.raffinosus, y E.avium raramente aislados de muestras clínicas pero que pueden poseer genes extracromosómicos que los hacen intrínsicamente resistentes a los glucopéptidos, los que podrían ser transferidos a E. faecalis y E. faecium. La colonización por estas bacterias es frecuente en pacientes gravemente enfermos ingresados en unidades de cuidado intensivo(UCI) y es un factor predisponente para septicemia. Los objetivos de este trabajo son la identificación de pacientes colonizados por enterococos vancomicina resistentes (EVR) en medios hospitalarios nacionales, el análisis de la sensibilidad a antibióticos de las bacterias aisladas, el estudio de los factores de riesgo para adquirirlas y de los aislamientos con alta resistencia a la vancomicina, con el fin de identificar determinantes genéticos de resistencia.

Pacientes y métodos: estudio prospectivo, que se desarrolló de mayo a agosto del año 2001. Se incluyeron 106 pacientes internados en las unidades de cuidado intensivo del Hospital México, Hospital San Juan de Dios y Servicio de emergencias médicas de ese último nosocomio, a los que se les tomaron hisopados rectales, los que fueron cultivados para identificar la presencia de EVR. Se determinaron las concentraciones mínimas inhibitorias (CIM) para vancomicina, y se realizó el estudio genético de las cepas con CIMs más altas.

Resultados: la tasa de colonización del tracto gastrointestinal de los pacientes fue de $52 \%$. Empleando las pruebas de chi cuadrado y de regresión logística, se identificaron factores que intervienen en la colonización por estas bacterias, siendo el servicio de procedencia antes del ingreso a las respectivas UCI, los días de estancia en UCI y el uso previo de cefalosporinas de tercera generación los principales.

El 29.6\% de los aislamientos de enterococos resistentes a vancomicina tenían CIM $\geq 512$ ug/ml, todos poseían el gen van A y correspondieron a E.gallinarum, E.faecium, E casseliflavus y E.hirae Todos los aislamientos de Enterococcus faecalis mostraron CIM $£ 16 \mathrm{ug} / \mathrm{ml}$ a la vancomicina.

Conclusiones: se demostró $52 \%$ de prevalencia de colonización en pacientes con EVR, superior a lo encontrado en otros estudios publicados en la literatura médica. Se establece la importancia de conocer estos hallazgos para el manejo clínico y epidemiológico de las infecciones asociadas a estas bacterias. Pocos estudios en la literatura mundial han logrado identificar este tipo de determinantes genéticos de resistencia (vanA) en enterococos no patogénicos. Se discute la relevancia de estos datos.

Descriptores: enterococos, enterococos vancomicina-resistentes, vancomicina, resistencia a antibióticos, antibióticos.

Recibido: 09 de setiembre de 2003

Aceptado: 17 de febrero de 2004 
Los enterococos son cocos Gram positivos, anteriormente clasificados dentro de los estreptococos pero que desde 1984 se consideran en un género aparte. ${ }^{1}$ Las especies de importancia médica son Enterococcus faecalis y E. faecium como productores de la mayoría de las infecciones en el ser humano y E.casseliflavus, E. gallinarum, E. durans, E.hirae, E.raffinosus, y E.avium raramente aislados de muestras clínicas y cuyo papel en procesos infecciosos en humanos es dudoso ${ }^{1}$, pero su importancia médica reside en que poseen genes extracromosómicos que las hacen intrínsicamente resistentes a los glucopéptidos los que podrían, en algunas circunstancias, ser transferidos a E. faecalis y E. faecium. ${ }^{2,3}$

Tradicionalmente los enterococos no han sido catalogados como microorganismos altamente virulentos, sin embargo son resistentes de manera intrínseca a una gran cantidad de antibióticos como clindamicina, cefalosporinas, sulfonamidas, trimetoprim, quinolonas y de forma adquirida a través de mutaciones o por transferencia de genes de resistencia de otras bacterias, a penicilinas (producción de b-lactamasas o de proteínas ligadoras de penicilinas de baja afinidad), tetraciclinas, eritromicina, rifampicina, cloranfenicol y glucopéptidos. ${ }^{1,2}$ La resistencia a estos últimos se ha identificado en determinantes genéticos van, de los cuales vanA y vanB, están ubicados en transposones, mientras que vanC, vanD, vanE, y vanG están localizados en el cromosoma y, aparentemente, no se transfieren horizontalmente. ${ }^{4-8} \mathrm{La}$ resistencia mediada por los deteminantes vanA y vanB confiere niveles de resistencia mayores que los otros determinantes. ${ }^{8}$

Se han identificado como agentes etiológicos de sepsis en el tracto urinario con alta frecuencia. ${ }^{1}$ Además han sido aislados a partir de infecciones de abdomen, pelvis, tracto biliar y heridas, sitios donde están presentes en ocasiones con una flora polimicrobiana. ${ }^{9-11} \mathrm{Se}$ les ha asociado a cuadros de meningitis y septicemia en neonatos y en adultos con historia de procedimientos neuroquirúrgicos o colocación de quimioterapia intratecal. ${ }^{1,2,10}$ Son causa de septicemia y endocarditis, infecciones con una alta mortalidad, especialmente en pacientes con antecedente de enfermedades oncohematológicas. ${ }^{10,11}$

Identificados por primera vez en 1986, los enterococos vancomicina-resistentes (EVR) han emergido en el ámbito mundial como importantes patógenos nosocomiales en los últimos diez años, en áreas como las unidades de cuidado intensivo (UCI), en servicios de hemato-oncología, unidades de transplante de médula ósea y en unidades de hemodiálisis. ${ }^{10-16}$ En 1999 en los Estados Unidos, de los cultivos positivos por enterococos, el $25 \%$ correspondió a EVR. ${ }^{12}$

La mayoría de los aislamientos de EVR corresponden a E.faecium, sin embargo se han notificado algunos aislamientos de E.faecalis, especie más frecuentemente aislada en infecciones en humanos. ${ }^{2,8-10}$

Ha sido demostrado que la colonización fecal por estas bacterias en pacientes con enfermedades onco-hematológicas o internados en UCI, es un factor de riesgo independiente para el desarrollo de septicemia por enterococos. ${ }^{13-16}$ Asimismo, la inmunosupresión, la neutropenia, la insuficiencia renal, el internamiento en UCI o en servicios de onco-hematología, la proximidad a un paciente colonizado con enterococos, el tiempo de estancia intrahospitalaria y el uso de antibióticos, son factores de riesgo para la colonización fecal por dichos gérmenes. ${ }^{10-16}$

En nuestro país ${ }^{17}$ se ha observado en los últimos años una mayor incidencia en el aislamiento de enterococos, principalmente E.faecalis, en pacientes atendidos en el Hospital San Juan de Dios, correspondiendo a un 6\% de las bacterias aisladas en tracto urinario y a un $9 \%$ de las aisladas en sangre, que aun cuando no han sido relacionados con resistencia a los glucopéptidos, se ha observado $25 \%$ de resistencia a la penicilina y hasta un $50 \%$ de resistencia a la gentamicina.

Por este motivo consideramos necesario iniciar un estudio de la prevalencia de estas bacterias en hisopados rectales en pacientes internados en servicios como las unidades de cuidado intensivo y emergencias médicas, en donde se tiene alta probabilidad de ser colonizados o de desarrollar procesos infecciosos por estos microorganismos. Asimismo es de gran importancia investigar la presencia de genes de resistencia a la vancomicina en estas bacterias, ya que incidirán tanto sobre la antibióticoterapia por emplear durante los procesos infecciosos que pudiesen desarrollar así como en el riesgo de la transmisión de los mismos a otros cocos Gram positivos.

Con los datos aportados en esta investigación, se podrán implementar medidas que disminuyan el riesgo de adquirir una infección por estos microorganismos.

\section{Población y métodos}

\section{Población}

Este es un estudio prospectivo, longitudinal y experimental realizado del 3 de mayo al 31 de agosto del año 2001, en las UCI del Hospital San Juan de Dios y Hospital México, que cuentan con 16 y 12 camas respectivamente. El estudio fue aprobado por el Comité de Ética y Científico del Hospital San Juan de Dios.

Se incluyeron pacientes que estaban ingresados en las UCIs respectivas y que aceptaron la toma del hisopado rectal. Los tiempos de estancia en estos servicios oscilaron entre 1 día y 103 días.

Se estudiaron también muestras de pacientes del Servicio de emergencias Médicas del Hospital San Juan de Dios, con una estancia intrahospitalaria de menos de 72 horas, que dieron su consentimiento para participar en el estudio. Este servicio tiene un mínimo de 14 camas para observación de pacientes. 
En total se recolectaron 141 muestras, las que fueron tomadas semanalmente en los servicios antes mencionados (30 muestras del Servicio de emergencias del Hospital San Juan de Dios, 76 de la unidad de cuidados intensivos del Hospital San Juan de Dios y 35 de la UCI del Hospital México), pero para este estudio solo se trabajó con las primeras muestras de los pacientes $(\mathrm{n}=106)$

\section{Métodos de laboratorio.}

El aislamiento de EVR a partir de las muestras de heces fue realizado siguiendo la metodología descrita anteriormente (18). Brevemente, los hisopados rectales fueron inmersos en $2 \mathrm{ml}$ de Agua Peptonada Bufferizada (peptona $10 \mathrm{~g} / \mathrm{l}$, $\mathrm{NaCl} 5 \mathrm{~g} / \mathrm{l}, \mathrm{Na} 2 \mathrm{HPO} 43.5 \mathrm{~g} /$, KH2PO4 $1.5 \mathrm{~g} / \mathrm{l}, \mathrm{pH} 7.2 \pm 0.2$ a $25^{\circ} \mathrm{C}$ ) e incubados a $35^{\circ} \mathrm{C}$ por $18-24$ horas. A partir del enriquecimiento se inocularon placas de agar infusión cerebro-corazón (Oxoid Ltd. Basingstoke, Hampshire, Inglaterra) conteniendo $0.04 \%(\mathrm{p} / \mathrm{v})$ de azida de sodio y $6 \mu \mathrm{g} / \mathrm{ml}$ de vancomicina. Las placas inoculadas se incubaron a $35^{\circ} \mathrm{C}$ con observaciones cada 24 horas hasta por tres días. Las colonias que correspondían a cocos Gram positivos catalasa-negativa fueron subcultivadas en agar sangre por $18-24$ horas a $35^{\circ} \mathrm{C}$. La identificación preliminar de los aislamientos se realizó mediante pruebas bioquímicas convencionales (crecimiento en $\mathrm{NaCl}$ al $6.5 \%$ y prueba de bilis-esculina positiva), como ha sido descrito. ${ }^{18,19}$ La identificación de algunos aislamientos fue confirmada mediante el sistema Vitek (bioMérieux, Marcy l'Etoile, France) con las tarjetas GPI, siguiendo las instrucciones del fabricante. La CIM a vancomicina se determinó mediante la técnica de dilución en agar. ${ }^{20}$

El aislamiento de ADN, la tipificación de los determinantes van y la determinación del fenotipo de alto nivel de resistencia (HLR) a aminoglicósidos (CIM gentamicina > $500 \mu \mathrm{g} / \mathrm{ml}$, CIM estreptomicina $>2,000 \mu \mathrm{g} / \mathrm{ml}$ ) se realizó siguiendo la metodología descrita previamente. ${ }^{18,21}$

\section{Análisis de los resultados.}

Una vez obtenidos los resultados de los cultivos y sus respectivas identificaciones, se clasificaron de acuerdo a las siguientes variables: edad, sexo, servicio y hospital de origen de la muestra, días de estancia intrahospitalaria, uso previo de antibióticos y duración de dichos tratamientos antes de la toma del hisopado rectal, servicio de procedencia antes de ingreso a la unidad de cuidado intensivo, antecedente de ingresos hospitalarios previos para los pacientes de emergencias, diagnósticos de ingreso y enfermedades concomitantes.

Posteriormente se procedió a establecer una correlación entre las variables antes mencionadas y el hecho de ser portador de EVR en tracto gastrointestinal, a través de la prueba estadística de chi cuadrado.

En algunos casos fue necesario aplicar el modelo de regresión logística, que permitió estimar la probabilidad de estar colonizado por EVR, en presencia de uno o más variables, al ser consideradas como posibles factores de riesgo.

\section{Resultados}

\section{Análisis descriptivo}

Características generales (cuadro 1). Los 30 pacientes de emergencias tenían una estancia intrahospitalaria de menos de 72 horas al momento de la recolección de la muestra y no tenían antecedentes de internamientos en los últimos 6 meses.

Servicios de procedencia al ingresar a la UCI. El 51.3\% de los pacientes provenía de servicios de medicina, siendo emergencias médicas el sitio de procedencia más frecuente, mientras que el $36.8 \%$ procedía de servicios de cirugía, el $6.6 \%$ de los servicios de gineco-obstetricia y $5.7 \%$ de otros servicios.

Uso previo de antibióticos. En 81 de los casos (77\%) se empleó al menos un tipo de antibiótico, 52 de ellos recibió dos antibióticos, 25 recibieron tres agentes antimicrobianos, siete recibieron cuatro y cinco pacientes recibieron hasta 5 antibióticos diferentes antes de la toma de la muestra. Los más utilizados fueron: aminoglucósidos, cefalosporinas de tercera generación, vancomicina y agentes antianaerobios

\begin{tabular}{|c|c|c|}
\hline \multicolumn{3}{|c|}{$\begin{array}{c}\text { Cuadro 1. Características generales de los } \\
\text { pacientes }(n=106)\end{array}$} \\
\hline \multicolumn{3}{|l|}{ Característica } \\
\hline Edad (años) & $14-71$ & × 54 \\
\hline \multirow[t]{2}{*}{ Sexo } & $50 \mathrm{H}$ & $47.2 \%$ \\
\hline & $56 \mathrm{M}$ & $52.8 \%$ \\
\hline \multirow{3}{*}{ Procedencia } & CI HSJD & $54(51 \%)$ \\
\hline & JCI HM & $22(21 \%)$ \\
\hline & nerg HSJD & $30(28 \%)$ \\
\hline \multicolumn{3}{|l|}{ Principales diagnósticos de ingreso } \\
\hline Neumonía & 30 & $28 \%$ \\
\hline Otras infecciones & 16 & $15 \%$ \\
\hline Cirugía cardiovascular & 13 & $12 \%$ \\
\hline Enfermedades neurológicas & 8 & $7.6 \%$ \\
\hline Politraumatismo & 6 & $5.7 \%$ \\
\hline Infarto agudo del miocardio & 6 & $5.7 \%$ \\
\hline $\begin{array}{l}\text { Otros (EPOC, ICC, DM } \\
\text { descomp, arritmias, hepatopatía) }\end{array}$ & 27 & $26 \%$ \\
\hline \multicolumn{3}{|c|}{ Principales enfermedades concomitantes } \\
\hline Diabetes mellitus & 25 & $23.6 \%$ \\
\hline Asma bronquial, EPOC & 18 & $17 \%$ \\
\hline Cardiopatía 18 17\% HTA & 17 & $16 \%$ \\
\hline Enfermedades hematológicas & 9 & $8.5 \%$ \\
\hline Sin enfermedades concomitantes & 24 & $22.6 \%$ \\
\hline \multicolumn{3}{|l|}{ Días de estancia en la UCI } \\
\hline$<5$ & 35 & $46 \%$ \\
\hline $5-10$ & 28 & $37 \%$ \\
\hline $11-15$ & 5 & $6 \%$ \\
\hline$>15$ & 8 & $11 \%$ \\
\hline \multicolumn{3}{|c|}{$\begin{array}{l}\text { UCI= unidad de cuidados intensivos; HSJD=Hospital San Juan } \\
\text { de Dios HM= Hospital México, Emerg HSJD =Servicio de emer- } \\
\text { gencias médicas, Hospital San Juan de Dios }\end{array}$} \\
\hline
\end{tabular}


(clindamicina y metronidazol). En promedio los pacientes habían recibido 5 días de antibióticoterapia al momento de la recolección de la muestra, para los diferentes tipos de antibióticos empleados.

\section{Aislamiento y genotipificación de EVR}

De los 106 pacientes estudiados, en 68 (64\%) se lograron aislar enterococos a partir de hisopados rectales y en 55 de ellos $(52 \%)$ se identificaron enterococos resistentes a vancomicina. En 13 pacientes (12\%) se aislaron enterococos vancomicina sensibles (EVS) y en $38(36 \%)$ no se aislaron enterococos.

De los 55 pacientes positivos por enterococos vancomicina-resistente se lograron detectar 83 aislamientos diferentes, basándose en características coloniales y en el perfil bioquímico. Estos aislamientos correspondieron a E. gallinarum $(\mathrm{n}=$ $35)$, E. casseliflavus $(n=24)$, E. faecium $(n=15)$, E. faecalis $(n=6)$, E. hirae $(n=1)$, Aerococcus $(n=1)$, mientras que un aislamiento no se pudo identificar. Se determinó una CIM a vancomicina $\geq 512 \mathrm{mg} / \mathrm{ml}$ en 24 de los aislamientos de Enterococcus vancomicina-resistente, los cuales eran todos portadores del gen vanA, y en el aislamiento de Aerococcus y el aislamiento no identificado. Así, los genes vanA y vanC se detectaron en el $28.9 \%$ y el $67.5 \%$ de los 81 aislamientos de Enterococcus vancomicina-resistentes analizados en este estudio. En gen vanA se logró detectar en diversas especies de Enterococcus, incluyendo E. gallinarum $(n=12)$, E. faecium $(n=10)$, E. casseliflavus $(n=1)$ y E. hirae $(n=1)$. No se pudo determinar el genotipo van en un aislamiento de E. gallinarum (CIM $4 \mathrm{mg} / \mathrm{ml}$ ), ni en el aislamiento de Aerococcus $(\mathrm{CIM}>512 \mathrm{mg} / \mathrm{ml})$, ni tampoco en el aislamiento no identificado (CIM $>512 \mathrm{mg} / \mathrm{ml}$ ). Estos dos últimos aislamientos presentan altos niveles de resistencia a vancomicina por un mecanismo independiente a los determinantes genéticos van.

De los 81 aislamientos de Enterococcus vancomicinaresistentes obtenidos de las muestras analizadas, $17(21 \%)$ y $9(11.1 \%)$ presentaron altos niveles de resistencia a gentamicina y estreptomicina, respectivamente. Trece de los 24 aislamientos van $\mathrm{A}+(54.2 \%)$ y 3 de los 56 aislamientos vanC+ $(5.4 \%)$ presentaron altos niveles de resistencia a gentamicina ( $\mathrm{p}<0.05)$. Por el contrario, 3 de los 24 aislamientos vanA+ (12.5\%) y 6 de los 56 aislamientos vanC+ (10.7\%) presentaron altos niveles de resistencia a estreptomicina $(\mathrm{p}>0.1)$. Así, el alto nivel de resistencia a gentamicina está asociado a la presencia del determinante genético vanA en los aislamientos de Enterococcus vancomicina-resistente analizados en este estudio.

\section{Análisis de correlaciones}

Para establecer las posibles correlaciones entre las variables analizadas y la colonización por EVR, se aplicó inicialmente la prueba de chi cuadrado.
No se comprobó asociación entre la edad, sexo, hospital de procedencia de los pacientes, diagnósticos de ingreso, enfermedades concomitantes y la colonización por estos gérmenes.

Para los pacientes de emergencias incluidos en el estudio $(n=30)$ no fue posible establecer una relación entre los ingresos hospitalarios previos, ni los días de estancia en el servicio de emergencias y la respectiva colonización por EVR, en este último caso la prueba se invalida por el número de pacientes de la muestra

En relación con el servicio de procedencia y los días de estancia en UCI, aplicando la prueba de chi cuadrado, se observó una asociación entre estas variables y la colonización por EVR.

Para poder establecer el tipo de relación se procedió a utilizar la regresión logística. El modelo establecido es estadísticamente significativo y clasifica correctamente el $71.05 \%$ de los casos observados.

Al analizar las posibilidades, se pudo interpretar que al estar un día más en la UCI, la relación de probabilidades de colonización por EVR con respecto a una persona que permanezca un día menos y manteniendo constante la procedencia del mismo servicio es 1.12, es decir, la probabilidad aumenta $12 \%$ por cada día más que se permanezca en la UCI, lo anterior es significativo estadísticamente con una $\mathrm{p}=0.0247$.

Con respecto a los servicios de procedencia, la probabilidad de ser portador de estos microorganismos cuando se procede de Emergencias es 7.3 veces la probabilidad de colonización que cuando se proceda de cirugía, 8.5 cuando se proviene de Salones de Medicina Interna y 6.19 a partir de salones de ginecobstetricia, con respecto a salones de cirugía.

Dicho de otra manera de acuerdo a los datos obtenidos, se comprobó que es menos probable colonizarse en salones de cirugía con respecto a emergencias, salones de medicina y ginecobstetricia (significativo al 1\%) (cuadro 2)

Se estudió también el efecto de los antibióticos sobre la colonización por EVR, según el tiempo de exposición al tratamiento.(cuadro 3)

Utilizando la prueba de chi cuadrado no se encontró correlación significativa entre el uso de antibióticos y la probabilidad de colonización, posiblemente por limitaciones propias de esta prueba por lo que se recurrió a la regresión logística.

Dicho modelo se empleó para el caso de los siguientes antibióticos: cefalosporinas de tercera generación, antibióticos antianaerobios y vancomicina.

Con respecto al uso de antibióticos antianaerobios (clindamicina y metronidazol), no se comprobó que se trate de un factor de riesgo para la colonización por EVR. 


\section{Cuadro 2. Distribución de los pacientes según servicio de procedencia y días de estancia en la $\mathrm{UCl}$ por colonización.( $\mathrm{n}=76)$}

\begin{tabular}{|lcccc|}
\hline Procedencia y estancia & $\begin{array}{c}\text { Colonizado } \\
\text { por EVR }\end{array}$ & $\begin{array}{c}\text { No } \\
\text { colonizado }\end{array}$ & Total & Significancia \\
\hline \multicolumn{1}{|c|}{ (N) } & 40 & 36 & 76 & \\
Servicio de procedencia & & & & $0,048 *$ \\
Emergencias Médicas & 14 & 8 & 22 & \\
Servicios de Medicina Interna & 11 & 6 & 17 & \\
Cirugía & 5 & 16 & 21 & \\
Emergencias Quirúrgicas & 5 & 2 & 7 & \\
Ginecobstetricia & 2 & 3 & 5 & \\
Otros & 3 & 1 & 4 & \\
Días de estancia en la UCI & & & & $0.054 * *$ \\
De 1 a menos de 5 días & 14 & 21 & 35 & \\
De 5 a menos de 10 días & 15 & 13 & 28 & \\
De 10 a menos de 15 días & 4 & 1 & 5 & \\
Más de 15 días & 7 & 1 & $8-$ & \\
\hline * Significativo al 5\% & & & & \\
** Significativo al 10\% & & & & \\
\hline
\end{tabular}

En el caso de las cefalosporinas de tercera generación, cuando éstas se emplean por 3 días o más, el riesgo de ser portador de dichos microorganismos en tracto gastrointestinal aumenta en casi 6 veces, comparado con los pacientes que no las reciben, sin embargo dicho dato no es estadísticamente significativo, por lo que no se puede afirmar que este sea un factor de riesgo.

En los pacientes que recibieron vancomicina por menos de 3 días se identificó un fenómeno interesante si se comparan con los individuos que no recibieron dicho antibiótico, ya que en los primeros existe un riesgo 8 veces mayor de estar colonizados por EVR, lo que es estadísticamente significativo.
Sin embargo cuando el individuo recibe vancomicina por más de tres días, dicha correlación pierde valor, disminuyendo en este último caso, el riesgo de ser portador de esas bacterias.

\section{D i s c u s i ón}

En este estudio se examinó la prevalencia de la colonización por EVR en el tracto gastrointestinal en pacientes de unidades de cuidado intensivo y emergencias, así como también los posibles factores de riesgo para la adquisición de estos microorganismos Además, se determinó la presencia de los determinantes van en los aislamientos obtenidos de los pacientes.

La tasa de colonización por EVR en el tracto gastrointestinal fue del $52 \%$, resultando más alta que la reportada en estudios previos. ${ }^{13-16,22-25}$

El $29.6 \%$ de los aislamientos de Enterococcus resistentes a vancomicina presentaron una CIM $\geq$ de $512 \mu \mathrm{g} / \mathrm{ml}$ y todos estos aislamientos eran portadores del gen vanA. Estos aislamientos incluyeron las especies E. faecium, Enterococcus gallinarum, E. casseliflavus y E. hirae. Se detectaron seis aislamientos de $\mathrm{E}$. faecalis pero todos presentaron CIM a vancomicina $£ 16 \mathrm{mg} / \mathrm{ml}$ y el gen vanC. La presencia de vanA en aislamientos de E. faecium es inquietante, ya que se han reportado como responsables de brotes en diversos hospitales en el mundo. ${ }^{2,3,10,11}$

La presencia del gen vanA, que confiere altos niveles de resistencia a vancomicina y teicoplanina, en E. gallinarum y E. casseliflavus ha sido reportada como poco frecuente, sin embargo desde 1994 ha sido descrita la transferencia de estos genes por conjugación a otros enterococos ${ }^{2,3}$, por lo que este hallazgo en el presente trabajo tiene enormes implicaciones clínicas y epidemiológicas. Aún más, en 3\% de los pacientes se aisló concomitantemente EVR y EVS. Recientemente

Cuadro 3. Colonización según tratamiento usado.

\begin{tabular}{|lccccc|}
\hline & $\begin{array}{c}\text { Colonizado } \\
\text { por EVR }\end{array}$ & $\begin{array}{c}\text { No } \\
\text { colonizado }\end{array}$ & $\begin{array}{c}\text { Posibilidad } \\
(\mathrm{OR})\end{array}$ & Intervalo & significancia** \\
\hline \multicolumn{1}{c}{$(\mathbf{N})$} & $\mathbf{4 0}$ & $\mathbf{3 6}$ & & & \\
Cefalosporinas 3 & & & & & \\
Generación & & & & & \\
No lo usan & 18 & 20 & Referencia & & \\
Menos de 3 días & 9 & 10 & 0.9015 & $0.27-3.01$ & 0.866 \\
3 días o más & 13 & 6 & 5.6606 & $0.69-46.5$ & 0.107 \\
Tratamiento & & & & & \\
Antianaerobios & & & & & \\
No lo usan & 30 & 30 & Referencia & & \\
Menos de 3 días & 3 & 3 & 1.3346 & $0.23-7.82$ & 0.749 \\
3 días o más & 7 & 3 & 0.7003 & $0.08-6.38$ & 0.752 \\
Tratamiento con & & & & & \\
Vancomicina & & & & & \\
No lo usan & 29 & 30 & Referencia & & \\
Menos de 3 días & 6 & 1 & 8.3747 & $0.92-75.9$ & 0.059 \\
3 días o más & 5 & 5 & 0.3469 & $0.05-2.37$ & 0.281 \\
\hline
\end{tabular}
Staphylococcus aureus aislado en una paciente, probablemente transferido a partir de una cepa de Enterococcus faecalis resistente a la vancomicina. ${ }^{26}$ Es la primera descripción in vivo de este fenómeno en la literatura mundial.

Se logró establecer una relación estadísticamente significativa entre el servicio de procedencia antes del ingreso a la UCI y el hecho de ser portador de estas bacterias en el tracto gastrointestinal, lo que apoya la idea de que los pacientes llegan ya colonizados a las unidades de cuidado intensivo; al respecto parece existir mayor riesgo de adquirir estos microorganismos en emergencias, salones de medicina y 
salones de ginecobstetricia, con respecto a los salones de cirugía, como ha sido demostrado en otras regiones ${ }^{13-15}$ relacionado a pacientes con mayores índices de co-morbilidad y más gravemente enfermos, así como también a mayores estancias intrahospitalarias y reingresos, sin olvidar las posibles diferencias con respecto a la higiene en el manejo de los pacientes y el hacinamiento que es una realidad en algunos servicios de nuestros hospitales.

Aunque algunos pacientes pudieron ingresar ya colonizados a las respectivas unidades de cuidado intensivo, al analizar los días de estancia y la posibilidad de colonizarse por estas bacterias, resultó una relación directa y estadísticamente significativa. El riesgo fue calculado y se observó que con cada día que se permanezca en la UCI el riesgo aumenta un $12 \%$. Este factor de riesgo ha sido demostrado en otros estudios. ${ }^{14-16}$ A diferencia de otros trabajos ${ }^{22-25}$ no se logró demostrar relación entre factores como edad, enfermedades concomitantes y diagnóstico de ingreso con la colonización por enterococos en UCI, lo que podría deberse al tamaño de la muestra. En un estudio previo sobre la presencia de EVR en muestras fecales de animales en Costa Rica, se logró detectar el determinante vanA en todos los aislamientos de E. durans, E. faecalis, E. faecium y E. hirae, en el 33.3\% de los aislamientos de E. gallinarum y en el $23 \%$ de los aislamientos de E. casseliflavus recuperados de muestras de aves de corral y cerdos. ${ }^{18}$ Así, diversas especies de Enterococcus vancomicina-resistentes portadoras del determinante genético vanA están circulando en ambientes extrahospitalarios en nuestro país. Sin embargo, la relación de los aislamientos animales con los aislamientos hospitalarios reportados en este estudio debe ser aún establecida.

Ha sido ampliamente estudiado el papel del uso previo de antibióticos sobre la colonización de los pacientes con EVR. Así las cefalosporinas de tercera generación, los antibióticos antianaerobios metronidazol y clindamicina y las fluoroquinolonas se han mostrado en diversos trabajos como factores directamente relacionados con este fenómeno. ${ }^{27-29}$

En el presente estudio, no se logró establecer relación entre el uso previo de agentes antianaerobios (metronidazol y clindamicina) y la condición de portador de EVR en el tracto gastrointestinal.

Con respecto al uso de cefalosporinas de tercera generación, el riesgo aumenta en casi 6 veces luego de recibir 5 días o más de tratamiento; este dato consideramos no debe ser despreciado, aunque la p no fue estadísticamente significativa, dicho resultado fue probablemente influenciado por el tamaño reducido de la muestra.

El uso de la vancomicina pareciera que no es un factor importante para la colonización por EVR. ${ }^{28}$ Esto ha sido explicado como un problema de diseño de los estudios realizados, ya que sí se ha encontrado relación con el uso de vancomicina para el desarrollo de septicemia. ${ }^{13}$
En este estudio se demostró que existe un riesgo mayor en pacientes que reciben vancomicina endovenosa por menos de tres días, sin embargo con el uso prolongado de este medicamento el riesgo disminuye, lo que puede explicarse por la selección de microorganismos aún más resistentes a la vancomicina, favoreciendo su crecimiento en comparación con los enterococos, tal es el caso de Pediococcus pentosaceus, Leuconostoc mesenteroides y Lactobacillus casei, éste último muy probablemente presente en el tracto gastrointestinal de estos pacientes, por el uso de yogurt como parte de la dieta que se les administra. Por todo lo anterior, deberán realizarse más estudios al respecto.

Es importante aclarar que los datos y observaciones no son aleatorias, ya que en su gran mayoría provienen de sujetos que dieron su consentimiento para la toma de la muestra, por lo tanto los resultados obtenidos no permiten hacer inferencia a la población general de hospitalizados, solo son válidas para las 106 observaciones.

Podemos concluir que la tasa de colonización por EVR en pacientes en este estudio fue más alta que la notificada en hospitales en Estados Unidos, Canadá y Europa , lo que podría deberse a malas normas de higiene, ya que bien sabemos que estas bacterias se transmiten por el mecanismo ano-manoboca o entre pacientes a través de las manos del personal de salud. ${ }^{1,2}$ Se demostró que la estancia intrahospitalaria y el uso de cefalosporinas de tercera generación son factores de riesgo independientes para la colonización por estos microorganismos, como ha sido previamente demostrado en otros estudio. Asimismo, como se ha demostrado en otras regiones, no está claro el papel del uso de la vancomicina y otros antibióticos en este fenómeno.

De resaltar fue el hallazgo de genes van A en enterococos usualmente no patogénicos y que transmiten altos niveles de resistencia a la vancomicina y a la teicoplanina, ya que éstos, como se dijo anteriormente, podrían ser traspasados a enterococos patógenos o a estafilococos.

A partir de la información obtenida con este trabajo, creemos necesario iniciar la conscientización del personal de salud, para implementar algunas medidas con el fin de reducir la transmisión nosocomial de EVR.

Han sido publicados varios estudios (30-34) para disminuir la diseminación de estas bacterias en el ambiente intrahospitalario, que se resumen de la siguiente manera:

1. Uso racional de antibióticos, especialmente el uso prudente de cefalosporinas de tercera generación, agentes antianaerobios y vancomicina. Se debe aprobar el uso de este último medicamento solo en caso de:

- Tratamiento de infecciones severas por microorganismos Gram positivos resistentes a antibióticos b-lactámicos. 
- Tratamiento de infecciones por microorganismos Gram positivos en pacientes con severas reacciones alérgicas a antibióticos b-lactámicos.

- Cuando la colitis asociada a antibióticos no responde al tratamiento con metronidazol y la condición del paciente es grave a causa de dicha enfermedad.

2. Profilaxis, como lo recomienda la Asociación Americana del Corazón, para endocarditis bacteriana en pacientes de alto riesgo y alérgicos a antibióticos b- Lactámicos. Profilaxis de cirugías mayores que involucren implantación de materiales protésicos como por ejemplo en procedimientos cardiovasculares, reemplazo total de cadera, en instituciones con alta incidencia de infecciones por $\mathrm{S}$. aureus o $\mathrm{S}$. epidermidis meticilina resistentes.

3. Programas educativos para el personal.

4. Vigilancia epidemiológica; realizando pruebas de sensibilidad a vancomicina a todas las cepas de enterococos que sean aisladas.

5. Identificación de pacientes con EVR al momento de readmisión.

6. Aislamiento de los pacientes en una habitación privada, aplicable a hospitales con baja y alta prevalencia de EVR.

7. Uso de guantes y batas para el manejo de los pacientes portadores.

8. Lavado e higiene de manos, una medida tan sencilla como esta es aplicable a todos los hospitales y puede reducir en forma importante la transmisión de estas bacterias entre pacientes, ya que se ha comprobado que las manos del personal de salud pueden ser un reservorio de estos microorganismos.

9. No se debe compartir equipo no crítico entre los pacientes como por ejemplo termómetros, esfigmomanómetros, estetoscopios, ya que los EVR pueden sobrevivir en estos objetos durante largos períodos de tiempo.

Es claro que estas medidas no requieren de grandes sacrificios o esfuerzos para el personal, ni para las diferentes instituciones de salud, de ahí la importancia de darlas a conocer e implementarlas en forma general en nuestros hospitales con el fin de prevenir y controlar las infecciones por estos gérmenes.

\section{A bstract}

Justification and Objectives: Enterococcus are Gram positive bacteria involved in community and nosocomial infections. There has been an increased interest in these bacteria because of their resistance to multiple antimicrobial drugs and their transposon-mediated resistance to glycopeptides.
Colonization of patients with enterococci is a risk factor for septicemia. Our main objectives were identification of patients colonized by vancomycin resistant enterococci (VRE) in two tertiary care hospitals, analysis of the antibiotic sensitivity to vancomycin of the isolated bacteria, study of the risk factors to acquire these bacteria and study of the isolations with high resistance profile to the vancomycin with the purpose of identifying genetic determinants of resistance, were the objectives of this study.

Patients and methods: this is a prospective, and experimental study conducted from may to august 2001 . We studied 106 patients hospitalized in the Intensive Care Units (ICU) of the Hospital México and Hospital San Juan de Dios and also the Medical Emergency Ward of the latter. Rectal swaps were taken in order to isolate VRE and to study the minimal inhibitory concentrations of vancomycin. Strains isolates with higher MIC's were studied for genetic determinants

Results: VRE colonization rates in gastrointestinal tract were $52 \%$. Using the chi square test and logistic regression some factors were identified for gastrointestinal tract colonization, being service of origin before admission to ICU, days of stay in ICU and previous use of third generation cephalosporins the main ones. VRE with MIC $\geq 512 \mathrm{mg} / \mathrm{ml}$ were demonstraded in $29.6 \%$ of the isolates, and in all of them (E.gallinarum, E.,faecium, E. casseliflavus, and E.hirae) the van A gen was found. All E. faecalis isolates showed vancomycin MIC£16 $\mathrm{mg} / \mathrm{ml}$.

Conclusions: We found a high colonization rate of the gastrointestinal tract, higher than the reported prevalence in other studies in the medical literature. Few studies in world wide literature have identified the van A gene in non-pathogenic enterococci. The relevance of these findings is discussed.

\section{Referencias}

1. Moellering RJ Enterococcus Species, Streptococcus bovis and Leuconostoc Species In Mandell, Douglas and Bennet's Principles and Practice of Infectious Diseases Fifth Edition New York Churchill Livingstone 2000;2147-2156

2. Gold H.S. Vancomycin-Resistant Enterococci: Mechanisms and Clinical Observations. Clin Infect Dis 2001; 33:210-219.

3. Dutka-Mallen S Blaimont B Wauters G Courvalin P Emergence of High-Level Resistance to Glycopeptides in Enterococcus gallinarum and Enterococcus casseliflavus Antimicrob Agents Chemother 1994-;38-:1675-1677

4. Fines M Perichon B Reynolds P Sahm D F Courvalin P VanE, a new type of acquired glycopeptide resistance in Enterococcus faecalis BM4405. Antimicrob Agents Chemother 1999; 43:2161-2164.

5. Gholizadeh Y Courvalin P Acquired and intrinsic glycopeptide resistance in enterococci. Int J Animicrob Agents 2000;16:S11-S17.

6. McKessar SJ Berry AM Bell JM Turnidge JD Paton JC Genetic characterization of vanG, a novel resistance locus of Enterococcus faecalis. Antimicrob. Agents Chemother 2000; 44:3234-3228. 
7. Perichon B Reynolds P Courvalin P. VanD type glycopeptide-resistant Enterococcus faecium BM4339. Antimicrob. Agents Chemother. 1997;41:2016-2018.

8. Cetinkaya Y Falk P Mayhall G Vancomycin-Resistant Enterococci Clin Microb Rev 2000;13:686-707

9. Moellering RJ Jr. Vancomycin-Resistant Enterococci. Clin Infect Dis 1998; 26:1196-1199

10. Rice LB Emergence of Vancomycin-Resistant Enterococci Emerg Infect Dis 2001;7: 183-187

11. Murray BE. Vancomycin-Resistant Enterococcal Infections. N Engl J Med. 2000; 342(10): 710-718.

12. Low DE Keller N Barth A Jones RN Clinical Prevalence, Antimicrobial Susceptibility, and Geographic Resistance Patterns of Enterococci: Results from the SENTRY Antimicrobial Surveillance Program, 19971999 Clin Infect Dis 2002;32(Suppl 2):S133-145

13. Zaas AK Song X Tucker P Perl TM Risk Factors for Development of Vancomycin-Resistant Enterococcal Bloodstream Infection in Patients with Cancer Who Are Colonized with Vancomycin-Resistant Enterococci Clin Infect Dis 2002; 35:1139-1146

14. Montecalvo MA deLancestre $H$ Carear $M$ Gedris $C$ Cheng $M$ van Horn K et al Natural history of colonization with vancomycin-resistant Enterococcus faecium. Infect Control Hosp Epidemiol 1995; $16: 680-685$

15. Gordst B van Landuyt H Leven M. van Damme P Goossens H Vancomycin-resistant enterococci colonizing the intestinal tracts of hospitalized patients. J Clin Microbiol 1995; 33: 2842-2846

16. Ostrowsky B Venkataraman L D'?Agata E Gold H DeGirolami P Samore M Vancomycin-Resistant Enterococci in Intensive Care Units. Arch Intern Med 1999; 159: 1467-1472.

17. Boza-Cordero R Barrantes-Valverde E Resistencia Bacteriana a Antibióticos en el Hospital San Juan de Dios 1995-1999 Acta Med Cost 2001;43:119-127

18. Bustamante W Alpízar A Hernández S Pacheco A Vargas N Herrera ML et al Predominance of vanA genotype among vancomycin-resistant Enterococcus isolates from poultry and swine in Costa Rica. Appl Environ Microbiol 2003; 69: 7414-7419.

19. Facklam RR Sahm DF Texeira LM Enterococcus In Murray PR Baron EJ Pfaller MF Tenover FC Yolken RH (eds) Manual of Clinical Microbiology 7th edition. American Society for Microbiology Press, Washington D. C. 1999; 297-305

20. National Committee for Clinical Laboratory Standards. Methods for dilution antimicrobial susceptibility tests for bacteria that grow aerobically. Approved standard M7-A4. National Committee for Clinical Laboratory Standards, Wayne, Pa. 1997

21. Free L Sahm DF Detection of enterococcal vancomycin resistance by multiplex PCR. In Persing DH (ed.) PCR protocols for emerging infectious diseases. American Society for Microbiology Press, Washington, D. C. $1996 ; 150-155$

22. van der Braak $N$ van Belkum $P$ van Keulen $M$ Vliegendhart J Prevalence and determinants of fecal colonization with VRE in hospitalized Netherlands patients. Infect Control Hosp Epidemiol 2000; 21:520524.

23. Karlowsky J A, Zhanel GG, Hoban DJ. VRE Colonization of High-Risk Patients in Tertiary Care Canadian Hospitals. Canadian VRE Surveillance Group. Diag Microbiol Infec Dis.1999; 35 : 1-7

24. Bonten MJM Slaugther S Amberger AW Hayden MK van Voorhis $\mathrm{J}$ Natham C et al The Role of Colonization Pressure in the Spread of Vancomycin-Resistant Enterococci Arch Intern Med 1998;158:11271132

25. Gambarotto K Prevalence of Vancomycin-Resistant Enterococci in Fecal Samples From Hospitalized Patients and Nonhospitalized Controls in a Cattle-Rearing Area of France J Clin Microb 2000;38:620624
26. Chang S Sievert DM Hageman JC Boulton ML Tenover FC Downers FP et al Infection with Vancomycin-Resistant Staphylococcus aureus Containing the van A Resistance Gene. N Engl J Med 2003; 348:13421347

27. Fridkin SK Edwards JR Courvalin JM Hill H Tenover FC Lawton R et al The Effect of Vancomycin and Third Generation Cephalosporins on Prevalence of Vancomycin-Resistant Enterococci in 126 US Adult Intensive Care Units. Ann Intern Med 2001;135:175-183

28. Carmeli Y Eliopoulos GM Samore MH Antecedent Treatment with Different Antibiotic Agents as a Risk Factor for Vancomycin-Resistant Enterococcus Emerg Infect Dis 2002; 8:802-807

29. Safdar N Maki D The Commonality of Risk Factors for Nosocomial Colonization and Infection with Antimicrobial-Resistant Staphylococcus aureus, Enterococcus, Gram-Negative Bacilli, Clostridium difficile and Candida Ann Intern Med 2002;136:834-844

30. Puzniak LA Mayfiel J Leet T Kollet M Mundy LM. Adquisition of Vancomycin-Resistant Enterococci during Scheduled Antimicrobial Rotation in an Intensive Care Unit. Clin Infect Dis 2000; 33:151-157

31. National Nosocomial Infectious Surveillance (NNIS) System Report, Data Summary from January 1990-May 1999 Hospital Infections Program, National Center for Infectious Diseases, CDC, Public Health Service, U.S Department of Health and Human Services, Atlanta, Georgia. June 1999

32. Goldmann DA Weinstein RA Wenzel RP. Strategies to prevent and control the emergence and spread of antimicrobial-resistant microorganisms in hospitals. A challenge to hospital leardership JAMA 1996; 275: $234-240$

33. Lautenbach E LaRosa L Marr AM Nachamkin I Bilker WB Fishman NO Changes in the Prevalence of Vancomycin-Resistant Enterococci in Response to Antimicrobial Formulary Interventions:Impact of Progressive Restrictions on Use of Vancomycin and Third -Generation Cephalosporins. Clin Infect Dis 2003;36:440-446

34. World Health Organization Antibiotic resistance: synthesis of recommendations by expert policy groups 2001 disponible en http://www. who.int/csr/resources/publications/drugresistance 